\title{
Diagnosis of listerial infections in infants
}

\author{
I. A. HARPER \\ C. H. L. Howells* \\ M.B., M.R.C.Path. \\ B.Sc., M.D., F.R.C.Path.
}

\section{Microbiology Laboratory, New Cross Hospital, Wolverhampton}

\section{Summary}

Five cases of suspected listerial infection are presented; four were proved to be genuine. Problems arose in the microscopy of smears of cerebrospinal fluid; the organism was not detected in one case, and in two other cases it was described as another type of bacterium. Difficulties in identifying the organism culturally are also discussed.

\section{Introduction}

Listerial infections have been reviewed extensively by Seeliger, Meyer \& Eyer (1961), Ray \& Wedgwood (1964), Barber \& Okubadejo (1965), Gray \& Killinger (1966), Voigt (1970) and others. Problems of identification and confusion with other organisms have been common, for example with diphtheroids (Barrow \& Pugh, 1958; Gray, 1960), Haemophilus influenzae (Moore \& Whitmore, 1960), Achromobacter spp. (Harding \& Brunton, 1962) and pneumococci (Graber, Higgins \& Davis, 1965). Ray \& Wedgwood (1964) cited difficulty with diphtheroids, atypical pneumococci, streptococci and Gramnegative bacilli.

In this communication we present four cases where cultural identification was made unequivocally, despite misleading initial microscopy on three of them and one case where a provisional initial diagnosis proved incorrect. Some helpful points in identification are also mentioned and the rarity of neonatal infections queried.

\section{Case histories}

\section{Case 1}

This baby was 3 weeks premature and at birth had nasopharyngeal brown discharge, rapid respirations and a purpuric generalized rash. $24 \mathrm{hr}$ later there was dyspnoea with head retraction and slight splenomegaly. Pyrexia $\left(39^{\circ} \mathrm{C}\right)$ began at this time and there was slight jaundice. The mother was a 40-yearold Jamaican with three other healthy children and a fourth had died when an infant. She had a normal pregnancy with no evidence of infection; there was no premature rupture of the membranes and the placenta was normal. She had intercourse 1 week before delivery; her husband was not investigated.

\footnotetext{
* Present address: Regional Public Health Laboratory, University Hospital of Wales, Heath Park, Cardiff.
}

A cord swab taken $48 \mathrm{hr}$ after delivery did not yield pathogens; cerebrospinal fluid (CSF) from the baby obtained 3 days after delivery contained an excess of polymorphonuclear leucocytes (WBC/ $R B C=\frac{1800}{1600} / \mathrm{mm}^{3}$ ) and a protein of $500 \mathrm{mg} / 100 \mathrm{ml}$. No micro-organisms were seen. However, L.monocytogenes was isolated after $24 \mathrm{hr}$ culture. The same organism was isolated from the infant's nose, throat and blood and also from the cervix and vagina of the mother.

Antibiotic therapy was started as soon as the diagnosis of pyogenic meningitis was made, penicillin, cephaloridine, hydrocortisone and sulphadiazine all being given i.m. Convulsions which began 4 days after delivery were controlled with difficulty using phenobarbitone and paraldehyde. Improvement was noted 3 days after treatment began and the baby was discharged after 3 more weeks apparently normal. The mother was treated with ampicillin as she appeared to be a carrier of the organism.

Although the infant's development was normal for 9 months, hydrocephalus was becoming apparent and lateral rectus palsy followed after a further 6 months.

\section{Case 2}

This baby was 8 days post-mature with some respiratory distress on delivery. 2 weeks later twitching of eyelids and rolling of eyes began. After a further week he was admitted to hospital with convulsions lasting $20 \mathrm{sec}$, occurring hourly. On examination there was cyanosis and a pyrexia of $39^{\circ} \mathrm{C}$, but no other localizing signs.

The mother had had an uncomplicated pregnancy and puerperium, without premature rupture of membranes, prolonged labour or pyrexia. There was one previous normal pregnancy.

Three days after admission, CSF was obtained from the baby and had an excess of polymorphonuclear leucocytes $\left(\mathrm{WBC} / \mathrm{RBC}=\frac{146}{670} / \mathrm{mm}^{3}\right)$ and a protein of $1500 \mathrm{mg} / 100 \mathrm{ml}$; bacilli, apparently Gram-negative, were seen. After $24 \mathrm{hr}$, L.monocytogenes was isolated. A cord swab 3 days after delivery did not yield any pathogens. It was not possible to investigate the mother.

The baby was treated with penicillin, chloram- 
phenicol and sulphadiazine, i.m. but died after a series of cardiac arrests.

At necropsy a purulent exudate extended over and below the cerebral hemispheres, in the subarachnoid space and into the ventricular system. Apart from marked pulmonary oedema, there were no other abnormalities. Swabs for culture were not taken.

\section{Case 3}

This child, aged 4 months, lived in a slum dwelling, occupied by twelve inhabitants, as well as numerous chickens, dogs and cats. He was admitted with a history of irritability and vomiting for 1 day, following 14 days of diarrhoea. There were no abnormal clinical signs except a pyrexia of $38.8^{\circ} \mathrm{C}$. Lumbar puncture yielded a turbid fluid with $298 \mathrm{WBC} / \mathrm{mm}^{3}$, $75 \%$ lymphocytes and a protein of $45 \mathrm{mg} / 100 \mathrm{ml}$. Gram-stained films showed scanty Gram-positive bacilli. On culture a pure growth of L.monocytogenes was obtained. The patient was treated with inthrathecal streptomycin, followed by i.m. chloramphenicol for 8 days and oral sulphadimidine for 6 days. Recovery was uneventful.

In an attempt to elucidate the source of infection, throat swabs, nasal swabs and blood cultures were taken from the father, three siblings and two friends, throat swabs, nasal swabs and vaginal swabs from the mother and two female lodgers. In addition, anal swabs were taken from a kitten, urine obtained from another kitten and faeces from a dog, puppy and chickens. L.monocytogenes was not isolated from any of these sources.

\section{Case 4}

This child was delivered by forceps and was one of twins, $5 \mathrm{lb}$ in weight at delivery. For 3 days before admission he had been off his feeds and was lethargic. There had been no vomiting or bowel disturbance. The other twin and the other members of the family remained well.

On examination the colour was good but the child appeared lethargic and somewhat wasted. Fluids were given and intravenous injections of ampicillin and penicillin were supported by intramuscular hydrocortisone and kanamycin. These measures were of no avail and the child died 2 days after admission.

Lumbar puncture on admission revealed yellow turbid fluid containing $1325 \mathrm{WBC} / \mathrm{mm}^{3}, 84 \%$ neutrophils. The protein was more than $200 \mathrm{mg} /$ $100 \mathrm{ml}$ rising to $1000 \mathrm{mg} / 100 \mathrm{ml}$ before death. A Gram-stained film of CSF revealed the presence of numerous Gram-positive cocco-bacilli reported as streptococci. L.monocytogenes was grown on culture from C.S.F. and blood.

There was no necropsy.
Case 5

This infant was the twin brother of the child mentioned in Case 4 (above). He was clinically well but a nasal swab was taken in the course of investigation of the family. On culture a Gram-positive rod was obtained with the bacteriological characteristics mentioned below.

\section{Bacteriological results}

The organisms isolated from the patients in Cases $1,2,3$ and 4 were similar in their characteristics. They grew on horse blood agar both aerobically and anaerobically at $24-48 \mathrm{hr}$, with a narrow zone of alpha haemolysis. They were motile, with a tumbling motion at room temperature and were non-motile or markedly less motile at $37^{\circ} \mathrm{C}$. They were catalasepositive and oxidase-negative. Glucose, trehalose, maltose and salicin were fermented. They did not ferment lactose or sucrose.

In Cases 1 (from both baby and mother), 2 and 4 the organism was sent to the Standards Laboratory for Serological Reagents, Colindale, and its identity was confirmed; the first two were both type 4 and that from Case 4 was type 1 . These strains had similar antibiotic sensitivity patterns and were alb sensitive, in a disc diffusion test in which zones were compared with those produced by the Oxfor staphylococcus, to penicillin, tetracycline, ampicillin cephaloridine, chloramphenicol and sulphonamide There was moderate resistance to streptomycin.

In Case 5, culture revealed a scanty growth of a Gram-positive rod which resembled listeria in its colonial characteristics (see above). It was weakly motile at room temperature and at $37^{\circ} \mathrm{C}$; the catalase reaction was difficult to define but seemed to be weakly positive. Sugar fermentations were in keeping with Listeria monocytogenes. The organism was excluded as a possible listeria by the Public Health Laboratory Service Reference Expert. It was later identified by the staff of the National Collection of Type Cultures as an organism closely allied to listeria for which a specific name was not available.

\section{Discussion and conclusions}

Reports of infection with Listeria monocytogenes are increasing in the U.K. (Barber \& Okubadejo, 1965; British Medical Journal, 1971) but are still relatively infrequent (British Medical Journal, 1969) as they are also in other countries (Becroft et al., 1971). The fact that we have diagnosed four proven cases in two Midland hospitals tends to support the view of others (Barrow \& Pugh, 1958; Moore \& Whitmore, 1960; Gray, 1960; Barber \& Okubadejo, $1965)$ that neonatal listerial infection at least is not as rare as is often supposed. Busch (1971) states that in the U.S.A. listeriosis in man is not rare but 
rarely recognized; he points out also that the incidence of human listeriosis there exceeds that of poliomyelitis, psittacosis or leptospirosis.

The potential seriousness of listeriosis is noted in a recent report (British Medical Journal, 1972) where a fatality rate of $20 \%$ is recorded over a 5 -year period.

In order to diagnose such infection however, as McKinnon \& Swithinbank (1961) emphasized, great care has to be taken both in the initial microscopy of films and in the inspection of cultures. The cases described here illustrate difficulties which may arise in both these areas. With regard to microscopy, it is important to remember that incorrect reporting of CSF may be a serious error as in listerial meningitis early antibiotic therapy is mandatory (Seeliger \& Matheis, 1969).

In Case 1 reported here, the organism was not observed and may have been present only in small numbers. In Case 2 it was reported as a Gramnegative bacillus and in Case 4 as a streptococcus. Only in Case 3 was the description correct. Thus, it is worth repeating the view that Gram-stained films of purulent CSF should always be checked, even at night, by someone of considerable bacteriological experience.

When cultural findings are considered there are two points to emphasize. First, any small alpha or beta haemolytic colony from a CSF culture should be examined most carefully. When the organism is a Gram-positive or weakly Gram-positive bacillus or a similarly staining pleomorphic organism and is catalase-positive then the possibility of its being Listeria monocytogenes should be considered. Tests of motility are extremely useful (see Case 5). Small volume broths (e.g. tryptose phosphate or glucose) should be inoculated as well as peptone water sugars to check for motility at $20-25^{\circ} \mathrm{C}$ and $37^{\circ} \mathrm{C}$ after 6,12 and $24 \mathrm{hr}$ incubation. If the organism is motile at $20-25^{\circ} \mathrm{C}$ and non-motile at $37^{\circ} \mathrm{C}$ it may be provisionally identified as Listeria monocytogenes. Confirmation by the finding of appropriate sugar fermentations is also necessary and may take a further $24 \mathrm{hr}$. Secondly, a diagnosis of listerial infection should be made only on adequate bacteriological evidence unless compelling clinical reasons exist, although in Case 5 in spite of the organism's doubtful motility it seemed reasonable to recommend antibiotic therapy in view of the isolation of listeria in a sibling. All the clinicians concerned favoured multiple antibiotic therapy in the absence of a bacteriological diagnosis and prescribed combinations of antibiotics to which Listeria monocytogenes is usually sensitive; in all these cases the antibiotic sensitivity tests confirmed the validity of the choice and were available within $24 \mathrm{hr}$ of isolation of the organism. The use of chloramphenicol was considered justifiable since the condition of the infants was critical, but it may be that ampicillin would have been of equal or greater value as suggested by Seeliger (1969).

Infections due to Listeria monocytogenes are not common and it is difficult to be dogmatic about antibiotic treatment. However, our impression is that ampicillin should now be the antibiotic of choice, preferably in combination with at least one other, e.g. cephaloridine or erythromycin; kanamycin in combination with ampicillin has been suggested also on the basis of in vitro studies (Louria et al., 1967; Gordon, Barrett \& Clark, 1972).

In the early stages of pyogenic meningitis of unknown aetiology where findings do not indicate the likely causal organism, we consider two possible regimens of treatment. The first is to use three antibiotics, penicillin, a sulphonamide and chloramphenicol; the second is to use only two, ampicillin and another antibiotic, for instance, cephaloridine (which will penetrate the CSF in meningitis) to obviate the use of chloramphenicol. However, we ourselves have not seen serious consequences of chloramphenicol treatment in cases of meningitis in infants.

It is preferable, wherever possible, to have the identity of an isolate confirmed by a Reference Laboratory and to have serological typing carried out. The latter step is essential if there is to be any advance in appreciating the epidemiology of listerial disease which is still poorly understood.

\section{Acknowledgments}

We should like to thank Miss Agnes Tannahill for the typing of isolates from Cases 1,2 and 4 and the National Collection of Type Cultures for the identification of the isolate from Case 5.

Our thanks are also due to Dr Michael Whitehead who encouraged one of us to pursue the findings in Cases 1 and 2, to the clinicians concerned for access to the case records and to the technical staff involved.

We are grateful to Sir James Howie for his helpful comments on the manuscript.

\section{References}

Barber, M. \& Okubadejo, O.A. (1965) Maternal and neonatal listeriosis: report of case and brief review of literature of listeriosis in man. British Medical Journal, 2, 735.

Barrow, G.I. \& PUGH, R.J. (1958) Listeria (erysipelothrix) monocytogenes meningitis in the newborn. Journal of Pathology and Bacteriology, 75, 9.

Becroft, D.M.O., Farmer, K., Seddon, R.J., Sowden, R., STEWART, J.H., Vines, A. \& WATTIE, D.ANN (1971). Epidemic listeriosis in the newborn. British Medical Journal, 3, 747.

British Medical Journal (1969) Epidemiology, listeriosis (based on reports to the Public Health Laboratory Service). 4, 751 .

British Medical Journal (1971) Epidemiology, listeriosis (based on reports to the Public Health Laboratory Service). 2, 477.

British Medical Journal (1972) Epidemiology, listeriosis (based on reports to the Public Health Laboratory Service). $2,415$. 
Busch, L.A. (1971) Human listeriosis in the United States (1967-69). Journal of Infectious Diseases, 123, 328.

Elston, H.R., ZenCKA, A.E., \& SKeTCH M.H. (1969) Listeria monocytogenes endocarditis. Archives of Internal Medicine, $124,488$.

Gordon, R.C., Barrett, F.F. \& Clark, D.J. (1972) Influence of several antibiotics, singly and in combination, on the growth of Listeria monocytogenes. Journal of Pediatrics, 80, 667.

Graber, C.D., Higgins, L.S. \& Davis, J.S. (1965) Seldomencountered agents of bacterial meningitis. Journal of the American Medical Association, 192, 956.

GRAY, M.L. (1960) Letter to Editor. Lancet, ii, 315.

Gray, M.L. \& Killinger, A.H. (1966) Listeria monocytogenes and listeric infections. Bacteriological Reviews, 30, 309.

HARding, J.W. \& BRUNTON, G.B. (1962) Listeria monocytogenes meningitis in neonates. Lancet, ii, 484.
Louria, D.B., Hensle, T., Armstrong, D., Collins, H.S., Blevins, A., Krugman, D. \& Buse, M. (1967) Listeriosis complicating malignant disease. A new association. Annals of International Medicine, 67, 261.

MCKinnon, D. \& Swithinbank, J.M. (1961) Meningitis due to Listeria monocytogenes. Lancet, ii, 516.

Moore, S. \& Whitmore, D.A. (1960) Case of listeria meningitis. British Medical Journal, 2, 1572.

RAY, C.C. \& Wedgwood, R.J. (1964) Neonatal listeriosis: six case reports and a review of the literature. Paediatrics, 34, 378.

SEeliger, H.P.R., Meyer, V.F. \& Eyer, H. (1961) Listeriosis. Hafner Publishing Co., New York.

Seeliger, H.P.R. \& Matheis, H. (1969) Treatment of human listeriosis. German Medical Monthly, 14, 566.

VoigT, J.C. (1970) Human listerial infection: four cases of meningitis and a review. British Journal of Clinical Practice, 24, 213. 